\title{
A Convenient Small Osmometer
}

\author{
G. A. Hanks and S. G. Weissberg
}

\begin{abstract}
A small, rugged osmometer that is relatively easy to assemble and use is described. Typical data obtained with polystyrene solutions are presented. Performance in comparison with other osmometers is discussed.
\end{abstract}

\section{Introduction}

A description is given of a simple, rugged instrument for determining the osmotic pressure developed by a solution of a high polymer. Other small osmometers described previously $[1,2,3,4,5],{ }^{1}$ have been, in some respects, too inconvenient to use. Although relatively easy to make, glass osmometers are fragile and may require frequent repairs. Small metal osmometers, in addition to being rugged, can be designed for easy and rapid assembly and simplicity of operation. The instrument described here is a modification of the apparatus described by Sands and Johnson [4]. The new design permits the instrument to be assembled, rinsed, and filled conveniently, while keeping the membrane wet with solvent at all times. The total volume of the solution cell is less than $2 \mathrm{ml}$ so that a relatively small quantity of polymer suffices for a complete osmotic pressure curve.

The Zimm and Myerson osmometer [5] has been used in the Bureau for some time. The outstanding advantages of this instrument are the ease of adjusting the solution meniscus and its relative speed in attaining equilibrium compared with other small osmometers. On the other hand, its assembly is tedious and time consuming. Particularly in rinsing the cell, it is difficult to empty the osmometer without risk of drying the membrane. After the instrument is finally filled with polymer solution, air bubbles must be removed from each osmometer. Because considerable clamping pressure on the membrane (which serves as its own gasket) is required to insure an adequate seal, there is danger that the glass edges may be chipped. This is especially likely if the plane of the membrane is not exactly perpendicular to the axis of the cylinder forming the cell of the Zimm osmometer.

\section{The Osmometer}

The osmometer design is shown in figure 1. The metal portion consists of an internally threaded brass member, a nickel-plated brass cell, an externally threaded brass member, and a perforated brass plate. The glass portion of the instrument consists of a measuring and a reference capillary, both cut from the same length of tubing of $0.5-\mathrm{mm}$ bore. A male standard taper (10/18) joint is sealed to one end of the measuring capillary. The reference capiliary is attached to the measuring capillary either by a

Figures in brackets indicate the literature references at the end of this paper. metal or glass bridge. A horizontal mark etched on the capillaries serves as a reference for capillary calibration. The male $10 / 18$ standard taper attached to the measuring capillary fits into the metal cell, which has been reamed and ground to accommodate it. A mercury well assures a leakproof seal. The flat surface of the cell adjacent to the membrane is lathe-finished and lapped on an optical lap to a mirror finish. The perforated plate adjacent to the other side of the membrane is lathe-finished only. The screw plug has three holes at $120^{\circ}$ intervals at the top. A wrench with three $1 / 4$-in. pins, which engage these holes, is used for final tightening of the assembly (fig. 2). For assembly purposes the osmometer base fits into a solvent well (fig. 2), in the floor of which are vertical dowel pins at $120^{\circ}$ intervals. These pins engage holes in the under side of $\mathrm{C}$. The base plate of the solvent well is attached to a channel iron that can be clamped to a work bench (fig. 2). A three-pronged brass hook (fig. 2) facilitates transferring the osmometer to and from the tubes that contain the solvent during the test. The hooks fit into the holes in $\mathrm{D}$.

\section{Assembly}

The assembly of this instrument is simple and rapid. The osmometer base is placed with the holes engaging the dowel pins in the solvent well. The perforated plate is fitted into the osmometer base. Solvent is then poured into the well up to about $0.5 \mathrm{~cm}$ above the level of the perforated plate. This assures that the membrane, which is put in place next, stays wet with solvent during the assembly period. The cell is next placed on the membrane. Finally, the screw plug is screwed in hand tight. The wrench is fitted into the holes on the screw plug, and a steel disk (fig. 2) is placed on top of the wrench. A C-clamp is used to clamp the assembly to the channel iron. This prevents the osmometer from riding upon the pins in the well during tightening. Final tightening is accomplished by using a 30 -in. extension to the wrench handle. The membrane, which serves as its own gasket, is thus squeezed between the perforated plate and the annular surface of the cell. A torque of approximately $800 \mathrm{in}$ - $\mathrm{lb}$ is sufficient to assure a leakproof seal. There is no evidence of shearing of the membrane during the tightening operation. The clamp is removed, and the osmometec is now ready to be filled with polymer solution. The capillaries should, in the meantime, have been cleaned with cleaning solution. 


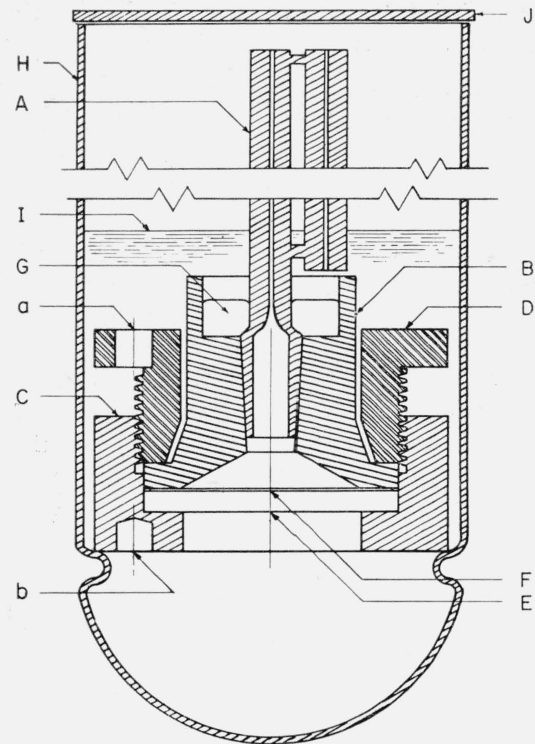

Figure 1. Assembled osmometer.

A, Solution and reference capillary; B, solution cell; C, osmometer base; D. pressure ring; $\mathrm{E}$, perforated plate; F, semipermeable membrane; G, mercury seal; H, solvent container; I, solvent level; J, cover plate; $a$, holes for wrench; $b$, holes engaging pins in assembly bath.
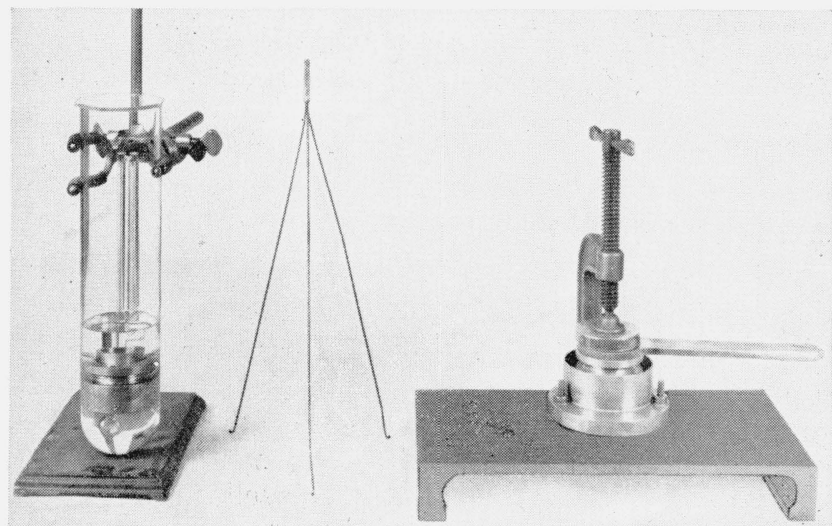

Figure 2. Assembled osmometer (left); three-pronged brass hook (center), and osmometer in position for tightening (right).

\section{Filling the Osmometer}

After pouring out the residual solvent, the cell is rinsed twice with the polymer solution and the perforations in $\mathrm{E}$ inspected. Any empty perforations may be filled by quickly inverting the assembly and adding solvent. The inversions must be performed quickly to prevent drainage of solvent from the holes in the perforated plate. The assembly is rinsed quickly a third time with solution and finally filled. The capillary assembly is then placed in position in the cell, but not firmly seated. Gentle suction is applied to the top of the measuring capillary until the meniscus level is visible near the base of the capillary assembly. The capillary assembly is then seated firmly. To prevent the solution from spilling over the top of the capillary, a clean absorbent towel is placed at the top to absorb excess solution. After firmly seating the capillary, excess solution is removed from the mercury well.

One must consider temperature effects in the initial adjustment of the solution meniscus. For static measurements, meniscus adjustment is not critical. The osmometer is placed in its upright position in the solvent well, after which the mercury well is flushed with solvent and dried. Approximately $2 \mathrm{ml}$ of clean distilled mercury is then placed in the mercury well. The assembly is removed from the solvent well, and the lower portion of the capillary and of the body proper are washed with solvent to remove residual polymer solution. The assembly is then transferred to the large test tube in which has been placed sufficient solvent for a convenient reference meniscus level. A small lateral hole cut through the base at a level just below the perforated plate permits the escape of air that might otherwise be trapped by the immersion. Air is removed by gently raising and lowering the osmometer assembly in the solvent. A small mirror placed under the large tube permits one to see when the trapped air has been completely removed. The osmometer assembly is now ready to be placed in the constant-temperature bath.

A revolving carriage in which 12 osmometers can be mounted in the constant-temperature bath permits each osmometer to be brought into position for measurements of the meniscus heights with a cathetometer.

\section{Membranes}

The following membranes have been used successfully in the National Bureau of Standards osmometers:

1. No. 600 Dupont cellophane (nonwaterproof variety). These membranes were conditioned according to the method of Carter and Record [6].

2. Never-dried regenerated cellulose (obtained from the Sylvania Corporation, Fredericksburg, Va). Conditioned according to the method of Fuoss and Mead [7].

3. Denitrated collodion membranes. In preparing these membranes, we generally followed the method of Montonna and Jilk [8]. It was found sufficient to evaporate solvent from the collodion very slowly overnight rather than to use the more elaborate method of Montonna and Jilk. Sufficient clean, distilled mercury is poured on a large (6 in.) petri dish to float a $12-\mathrm{cm}$ (id) chrome-plated iron ring. Into the ring are poured 37 to $40 \mathrm{ml}$ of collodion (free from plasticizer). The lid is placed on the petri dish, and the whole assembly is left in a dust-free box overnight under slight vacuum to remove solvent vapors. After overnight evaporation of solvent, the collodion film is submerged in distilled water to loosen the film from the ring. The ridge is immediately cut from the edge of the film, after which the sheet is placed in distilled water until final traces of solvent are removed. The film is then denitrated, following Montonna and Jilk's technique. A small glass stirring rod attached to an air-driven motor is used to agitate the ammonium sulfide solution during the denitration procedure. 
Membranes after denitration can be stored in distilled water to which has been added a little formalin to prevent bacterial action on the film. A more satisfactory method of membrane storage is to condition freshly prepared membranes to acetone. Water is displaced from the sheets by washing them in successive acetone-water mixture of increasing acetone concentrations, and the sheets are finally stored under distilled water-free acetone until ready for use.

\section{Performance}

A comparison of the relative speeds in attainment of osmotic equilibrium for the NBS and the Zimm osmometers was made with a solution of polystyrene in toluene. ${ }^{2}$ The results of several measurements are shown in figure 3. The ratio of the total membrane area of the Zimm to NBS osmometers is approximately 3.8 and of the solution volumes is 5.7. The ratio of the rates of approach to equilibrium of the Zimm to NBS osmometers was 3.2. The membranes in these osmometers were probably nearly equal in their permeability to solvent because they were cut from the same sheet and were prepared under identical experimental conditions. It is apparent that the Zimm osmometer is faster than the NBS osmometer, the extra membrane area and the vertical orientation of the membranes more than compensating for the difference in volume. The vertical membranes facilitate mixing of solvent and solution by convection, whereas the horizontal membranes permit convection only when the approach to osmotic equilibrium is from lower to higher pressures.

Fluctuations with time of the final equilibrium osmotic pressures amount to approximately \pm 0.02 $\mathrm{cm}$. This can be attributed to bath-temperature variations. "Membrane asymmetry corrections" were of the same magnitude. Because of these small differences in the osmotic pressures, no corrections
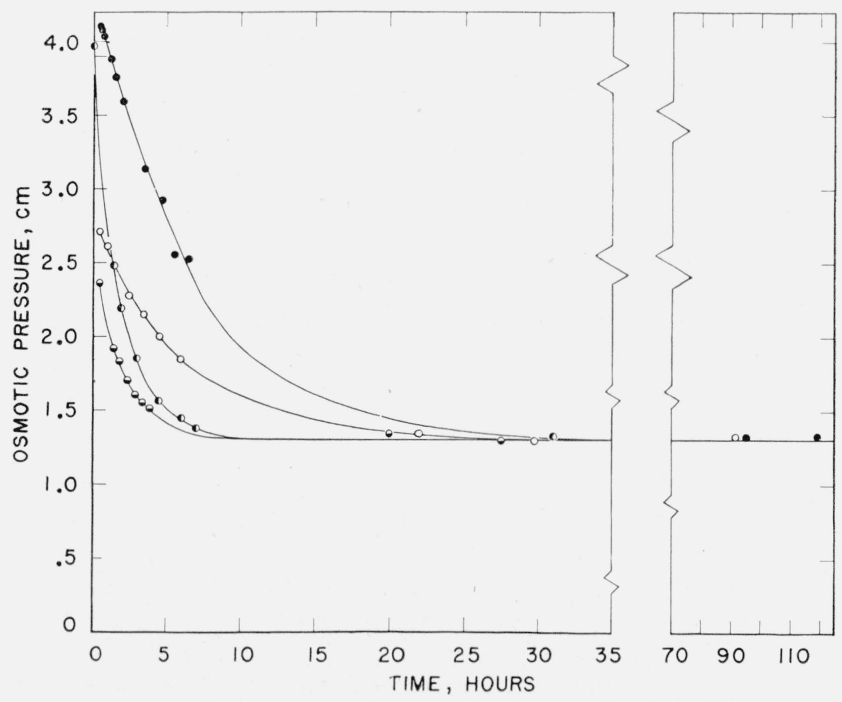

Figure 3. Rate curves obtained with National Bureau of Standards and Zimm types of osmometers.

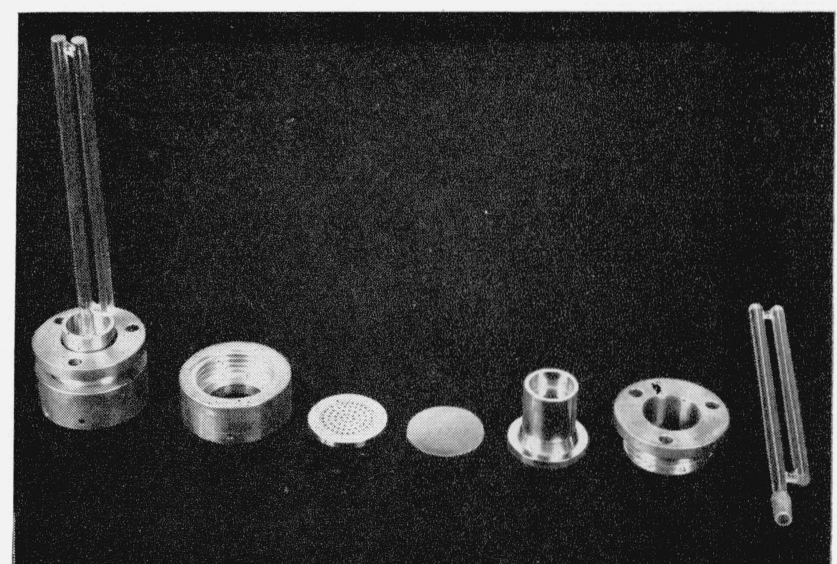

Figure 4. Osmometer, assembled and disassembled.

TABLE 1. Reproducibility of results obtained with the NBS osmometer

Polystyrene fractions in toluene

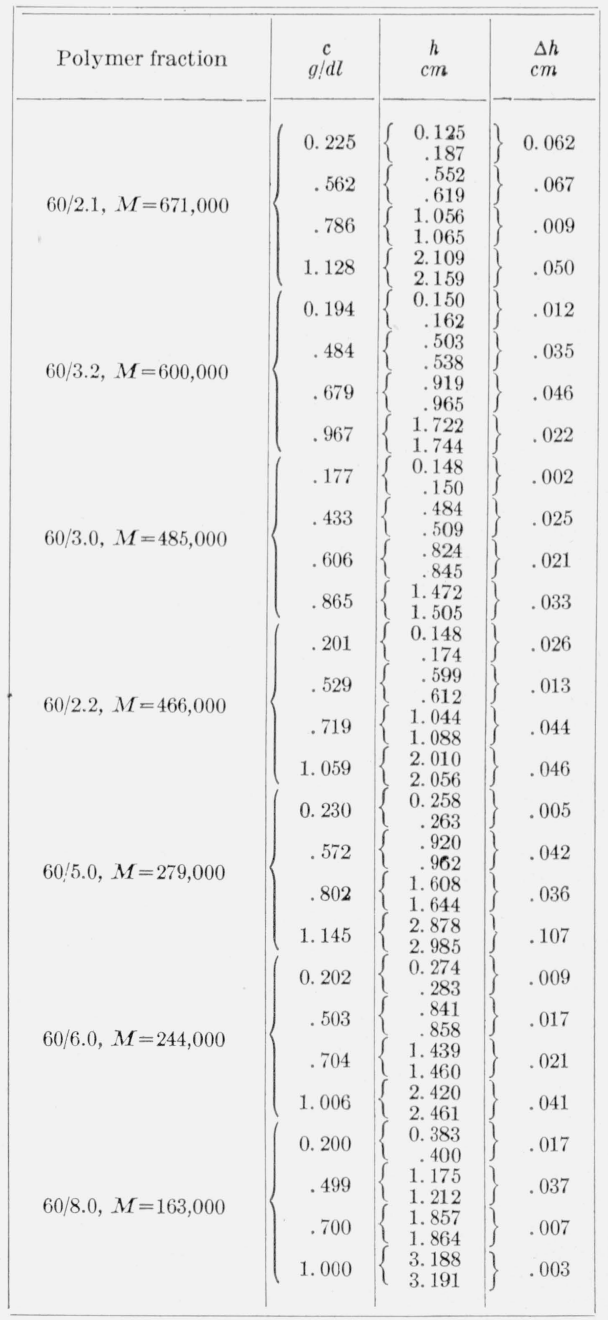

${ }_{2}$ These fractions were prepared from a polystyrene bulk polymerized without catalyst at $60^{\circ} \mathrm{C}$ by differential precipitation from butanone solution, using methanol as precipitant. Fractions designated by $X .0$ are derived from a single precipitation; a fraction designated by $X . Y$ is the $Y^{\text {tb }}$ subfraction of fraction $\mathrm{X} .0$. 
for membrane asymmetry were made. Table 1 shows the duplicability of results with NBS osmometers for polystyrene fractions in toluene solution. ${ }^{3}$ Figure 4 shows an assembled osmometer and parts of the completely disassembled instrument.

The instrument has been successfully used by P. J. Flory and associates $[9,10,11]$.

\section{Discussion}

Advantages of the osmometer are:

1. Rugged and simple construction.

2. Rapid assembly, rinsing, and filling of cell. Assembly and filling time is approximately six times as fast with the NBS osmometer as with the Zimm instrument.

3. Minimum possibility of membrane drying.

4. Small volume of cell $(1.2 \mathrm{ml})$.

5. Can be adequately tightened (membrane between perforated plate and lapped surface of cell), especially when using hydrocarbon solvents, which cause considerable membrane shrinkage.

6. Reduced possibility of entrapment of air in cell.

7. The complete osmometer assembly is small, hence a number of them can be used simultaneously for a complete osmotic pressure determination.

8. Acme thread on this instrument has pressure angle of $29.5^{\circ}$, a better pressure angle than the $\mathrm{V}$-type thread, whose angle is $60^{\circ}$.

9. Ease of soaking cell in large volume of solvent to minimize adsorption of polymer.

Disadvantages of the NBS osmometer are:

1. Precise meniscus adjustment is more difficult than with the Zimm osmometer. However, this is not critical when the instrument is being used for static equilibrium measurements.

2. Two to three days are sometimes necessary for osmotic equilibrium to develop, although this will depend primarily on the initial head and permea-

\footnotetext{
3 See footnote 2 .
}

bility of the membrane. The use of a horizontal membrane permits equalization of concentration only by diffusion when the osmotic equilibrium is approached from above. Osmometers with membranes in a vertical plane have, in addition, convective mixing.

It has been the experience here that of the three types of membranes used for osmotic-pressure measurements, denitrated collodion is generally superior in performance. Never-dried cellulose is a "faster" membrane but is permeable to low molecular weight polymer particles. Number 600 Dupont cellophane is "slower" than denitrated collodion but can be used satisfactorily when determining the osmotic pressure of low molecular weight polymers.

3. Occasional membrane buckling makes it difficult to make dynamic measurements, as is the case of any osmometer that supports the membrane on only one side.

\section{References}

[1] F. T. Wall, F. W. Banes, and G. D. Sands, J. Am. Chem. Soc. 68, 1429 (1946).

[2] D. M. French and R. H. Ewart, Ind. Eng. Chem., Anal. Ed. 19, 165 (1947)

[3] R. H. Wagner, Ind. Eng. Chem., Anal. Ed. 16, 520 (1944).

[4] G. D. Sands and B. L. Johnson, Ind. Eng. Chem., Anal. Ed. 19, 261 (1947).

[5] B. H. Zimm and I. Myerson, J. Am. Chem. Soc. 68, 911 (1946).

[6] S. R. Carter and B. R. Record, J. Chem. Soc. p. 660 (1939)

[7] R. M. Fuoss and D. J. Mead, J. Phys. Chem. 47, 59 (1943).

[8] R. E. Montonna and L. T. Jilk, J. Phys. Chem. 45, 1374 (1941).

[9] H. L. Wagner and P. J. Flory, J. Am. Chem. Soc. 74, 195 (1952).

[10] L. Mandelkern and P. J. Flory, J. Am. Chem. Soc. 74, $2517(1952)$

[11] P. J. Flory, L. Mandelkern, J. B. Kinsinger, and W. B. Schultz, J. Am. Chem. Soc. 74, 3364 (1952).

Washington, September 10, 1952. 